\title{
Paliperidone Palmitate-Induced Delirium in an Adolescent with Schizophrenia Case report
}

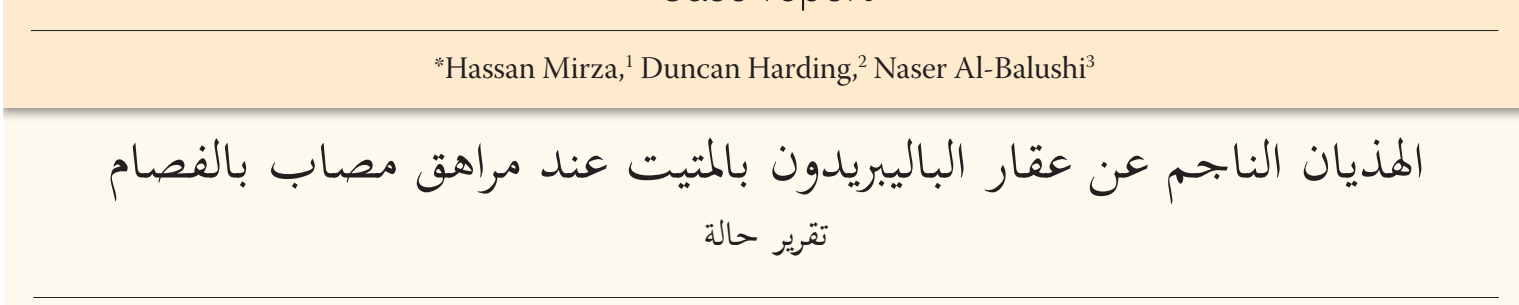

$$
\text { حسن ميرزا، دنكن هاردينج، نصر البلوشي }
$$

ABSTRACT: Schizophrenia is a serious long-term mental disorder which usually presents in adolescence or early adulthood. However, poor adherence to oral antipsychotics can lead to relapse and rehospitalisation. We report an adolescent male with schizophrenia who was referred to the South London \& Maudsley National Health Service Foundation Trust, London, UK, in 2015 due to worsening psychotic symptoms. Following poor compliance with oral medications, a four-week regimen of paliperidone palmitate long-acting injections was initiated, with an initial positive response. However, 10 days after the second dose, the patient developed severe acute-onset delirium with fluctuating levels of consciousness. Paliperidone palmitate was discontinued and the patient instead underwent a course of zuclopenthixol decanoate long-acting injections with a favourable outcome.

Keywords: Adolescent Psychiatry; Schizophrenia; Antipsychotic Agents; Delirium; Paliperidone Palmitate; Zuclopenthixol; Case Report; United Kingdom.

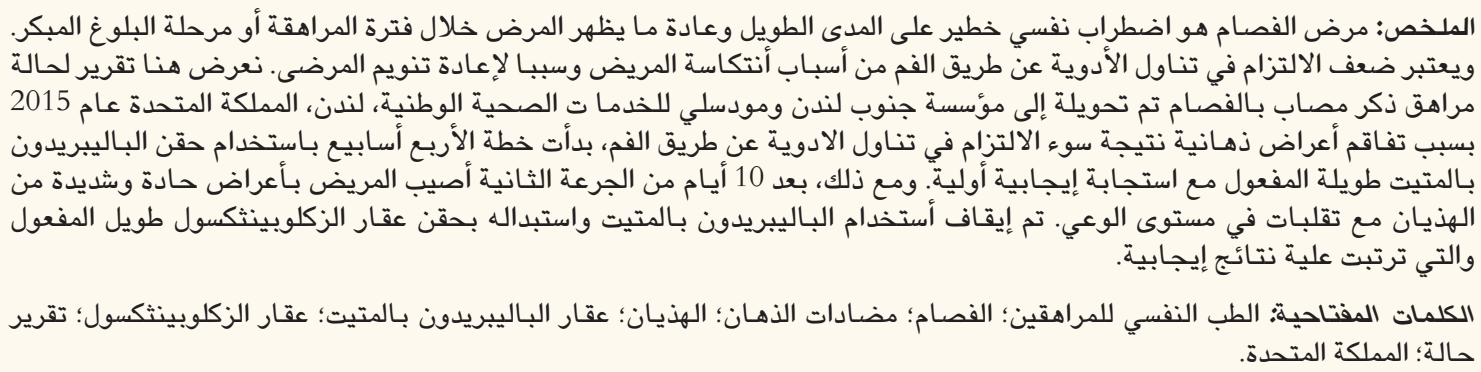

S CHIZOPHRENIA IS A SERIOUS MENTAL DISORDER characterised by the distortion of thought, perception and affect. ${ }^{1}$ Overall, the disease has a massive global burden, with a lifetime prevalence of four cases per 1,000 individuals. ${ }^{2,3}$ In younger patients, extra care needs to be taken when prescribing antipsychotic medications, since such individuals are at higher risk of developing side-effects. ${ }^{4}$ In general, second-generation antipsychotics (SGAs) are the first line of treatment for children and adolescents, whereas first-generation antipsychotics (FGAs) should be avoided due to the high risk of extrapyramidal sideeffects. ${ }^{5}$ Nevertheless, adherence to oral antipsychotic medications remains an ongoing challenge among patients with schizophrenia; the administration of medication in the form of a long-acting injection (LAI) can help overcome this issue, thus reducing the chance of relapse and rehospitalisation. ${ }^{6}$

\section{Case Report}

A 16-year-old Caucasian male patient was referred by his general practitioner to the Child \& Adolescent Mental Health Services of the South London \& Maudsley National Health Service Foundation Trust, London, UK, in 2015, following the worsening of existing psychotic symptoms. The patient had a oneyear history of low mood, anxiety, paranoia and social isolation. These symptoms initially presented solely in the context of cannabis use; however, the patient had stopped smoking cannabis when he noticed that this worsened his distress. Psychiatrically, he exhibited all the hallmarks of paranoid ideation. He believed that his room was being monitored and described auditory command hallucinations in which voices would tell him to get dressed in the middle of the night and go outside to walk in the park, as well as 
making derogatory remarks about him. The command hallucinations overpowered his usual distress of leaving his house, but did not tell him to harm himself or others. He also described elements of thought interference-i.e. thoughts being taken from and put into his head-and worried that his thoughts were being broadcast to others.

The patient had a strong family history of mental disorders, with a history of depression in his mother and two half-siblings who suffered from psychotic illness. Regarding his personal history, he had achieved normal developmental milestones and attended primary school; however, he had been excluded from secondary school following repeated altercations with his teachers. He had no friends and led a reclusive lifestyle. His social functioning had deteriorated significantly over the last year and even more drastically in the weeks prior to his referral. He reported feelings of low mood, anxiety, anhedonia and difficulty falling asleep.

Upon referral, the patient was initially prescribed oral olanzapine at a dosage of $10 \mathrm{mg}$ once daily, which was titrated up to $20 \mathrm{mg}$ per day. However, due to poor compliance with oral medications and the persistence of his psychotic symptoms, a regimen of olanzapine LAIs at a dose of $300 \mathrm{mg}$ every two weeks was initiated. Although he demonstrated a reasonable response to the depot injections, these had to be discontinued due to the unavailability of a post-injection observation service and the lack of transport provision. Subsequently, the patient was prescribed oral risperidone for approximately two months, after which risperidone LAI was commenced. The dose of the depot injection was titrated to $50 \mathrm{mg}$ every two weeks. The patient showed a moderate response to the treatment, although he was only partially compliant, often refusing injections and not attending his appointments every two weeks. As a result, the LAI treatment was also discontinued and the patient was again prescribed oral risperidone. This oral regimen was continued for approximately one month. The option of a monthly LAI formulation was then discussed with the patient and his caretaker. Eventually, a regimen of paliperidone palmitate LAIs every four weeks was agreed upon.

Based on his body mass index and medium build, the patient was prescribed off-label paliperidone palmitate LAIs, as per the manufacturer's instructions and the recommendations of the British National Formulary for adult patients, at an initial loading dose of $150 \mathrm{mg}$, followed by $100 \mathrm{mg}$ on the $8^{\text {th }}$ day. ${ }^{7}$ Both the patient and his mother reported an initial positive response to the medication, with a marked reduction in distressing psychotic symptoms. However, 10 days after the second dose, the patient developed acuteonset altered sensorium and was admitted to the Accident \& Emergency Department. Prior to this, the patient had been unable to sleep and reported unusual perceptual experiences such as hearing thunder and storms and smelling an unpleasant odour like burning tyres. He also suffered from fluctuating levels of consciousness, disordered speech with 'snort'-like breathing and flailing movements of the extremities.

Neurological impairment was minor, with a Glasgow Coma Scale score of 13-15. There were no frank convulsions and the patient was afebrile and did not report any headaches. He did not show any evidence of autonomic system imbalance and there was no rigidity or other features indicative of neuroleptic malignant syndrome. His C-reactive protein and serum creatine kinase levels were normal and a computed tomography scan of the brain, comprehensive metabolic panel, liver function tests, urinalysis and urine toxicology screening were unremarkable. A full blood count initially showed mildly raised leukocytes; however, when repeated the next day, all parameters were within normal ranges, with no signs indicative of a focal or systemic infection.

By the evening of the next day, the patient had regained consciousness and was found to be fully alert and awake. He could not recall any of the events of the previous day. He was subsequently discharged against medical advice and returned home to the care of his mother. Since the patient continued to experience both positive and negative schizophrenic symptoms, zuclopenthixol decanoate injections were prescribed at an initial test dose of $100 \mathrm{mg}$, before eventually being titrated to $200 \mathrm{mg}$ monthly. After three months of treatment, there was a significant improvement in psychotic symptoms. At the time of the writing, the patient had not reported any serious side-effects and his activities of daily living had reverted to their premorbid status.

\section{Discussion}

The present case report describes an adolescent with schizophrenia who developed postinjection delirium/ sedation syndrome (PDSS) following a regimen of paliperidone palmitate LAI. To the best of the authors' knowledge, few similar cases have been reported in the literature. Kokalj et al. reported a female schizophrenic patient who became delirious following an olanzapine LAI and a higher dose of paliperidone palmitate; as with the current case, the patient had a history of cannabis use. ${ }^{8}$

Paliperidone palmitate injections differ from other antipsychotic depot injections in that two loading 
doses are required at the start of treatment, consisting of $150 \mathrm{mg}$ on day one and $100 \mathrm{mg}$ on day eight; these are then adjusted at monthly intervals according to the patient's response, with a recommended maintenance dose of $75 \mathrm{mg}$ per month (range: $25-150 \mathrm{mg}$ ). ${ }^{7}$ Although no long-acting antipsychotic injections are yet officially recommended for individuals under the age of 18 years, their use is not uncommon in patients with a history of poor medication adherence. ${ }^{9}$

Paliperidone palmitate is deemed an effective and acceptably tolerated antipsychotic in adolescents with psychotic spectrum disorders. ${ }^{10}$ In a review of clinical trial databases, Alphs et al. found no cases of PDSS reported in completed trials of risperidone LAI, with only one PDSS case reported in a paliperidone palmitate LAI trial; however, the patient in this instance had been randomly allocated to the placebo treatment. ${ }^{11}$ Nevertheless, manufacturers of paliperidone palmitate LAI have acknowledged that a confusional state is a possible, albeit rare, adverse reaction to the drug. ${ }^{12}$ Although SGAs have been increasingly used as the first line of treatment for schizophrenia over the past decade, several studies have suggested there is limited or no advantage between the two classes of antipsychotics in terms of efficacy, with the only difference being the drug side-effect profile. ${ }^{13,14}$ Similarly, despite their limited use, FGAs remain an option in the management of psychotic disorders among adolescents, as in the current case wherein the patient showed a good response and tolerability to zuclopenthixol decanoate. ${ }^{15,16}$

\section{Conclusion}

Schizophrenic patients and their caretakers should be made aware that PDSS is an uncommon sideeffect of SGAs such as paliperidone palmitate. This case illustrates the importance of patient and family education regarding the potential side-effects of antipsychotic LAIs.

\section{References}

1. World Health Organisation. The ICD-10 Classification of Mental and Behavioural Disorders: Clinical descriptions and diagnostic guidelines - Tenth revision of the International Statistical Classification of Diseases and Related Health Problems. From: http://apps.who.int/iris/handle/10665/37958 Accessed: Jan 2018.

2. Bhugra D. The global prevalence of schizophrenia. PLoS Med 2005; 2:e151. doi: 10.1371/journal.pmed.0020151.
3. Saha S, Chant D, Welham J, McGrath J. A systematic review of the prevalence of schizophrenia. PLoS Med 2005; 2:e141. doi: 10.1371/journal.pmed.0020141.

4. Correll CU. Addressing adverse effects of antipsychotic treatment in young patients with schizophrenia. J Clin Psychiatry 2011; 72:e01. doi: 10.4088/JCP.9101tx6c.

5. Taylor D, Paton C, Kapur S. Children and adolescents. In: The Maudsley Prescribing Guidelines in Psychiatry, 12th ed. Hoboken, New Jersey, USA: Wiley-Blackwell, 2015. Pp. 353-408.

6. Chue P. Long-acting risperidone injection: Efficacy, safety, and cost-effectiveness of the first long-acting atypical antipsychotic. Neuropsychiatr Dis Treat 2007; 3:13-39. doi: 10.2147/nedt.2007. 3.1.13.

7. British National Formulary. BNF 70: September 2015 - March 2016. From: https://pharm.reviews/images/statyi/british-national-for mulary-2015.pdf Accessed: Jan 2018.

8. Kokalj A, Rijavec N, Tavčar R. Delirium with anticholinergic symptoms after a combination of paliperidone and olanzapine pamoate in a patient known to smoke cannabis: An unfortunate coincidence. BMJ Case Rep 2016; 2016:bcr2016214806. doi: 10. 1136/bcr-2016-214806.

9. McClellan J, Stock S; American Academy of Child and Adolescent Psychiatry (AACAP) Committee on Quality Issues (CQI). Practice parameter for the assessment and treatment of children and adolescents with schizophrenia. J Am Acad Child Adolesc Psychiatry 2013; 52:976-90. doi: 10.1016/j.jaac.2013.02.008.

10. Fàbrega M, Sugranyes G, Baeza I. Two cases of long-acting paliperidone in adolescence. Ther Adv Psychopharmacol 2015; 5:304-6. doi: 10.1177/2045125315600141.

11. Alphs L, Gopal S, Karcher K, Kent J, Sliwa JK, Kushner S, et al. Are the long-acting intramuscular formulations of risperidone or paliperidone palmitate associated with post-injection delirium/ sedation syndrome? An assessment of safety databases. Curr Drug Saf 2011; 6:43-5. doi: 10.2174/157488611794480070.

12. European Medicines Agency. Xeplion. From: www.medicines. org.uk/emc/files/pil.7652.pdf Accessed: Jan 2018.

13. Jones PB, Barnes TR, Davies L, Dunn G, Lloyd H, Hayhurst KP, et al. Randomized controlled trial of the effect on quality of life of second- vs first-generation antipsychotic drugs in schizophrenia: Cost Utility of the Latest Antipsychotic Drugs in Schizophrenia Study (CUtLASS 1). Arch Gen Psychiatry 2006; 63:1079-87. doi: 10.1001/archpsyc.63.10.1079.

14. Crossley NA, Constante M, McGuire P, Power P. Efficacy of atypical v. typical antipsychotics in the treatment of early psychosis: Meta-analysis. Br J Psychiatry 2010; 196:434-9. doi: 10.1192/bjp.bp.109.066217.

15. Pope S, Zaraa SG. Efficacy of long-acting injectable antipsychotics in adolescents. J Child Adolesc Psychopharmacol 2016; 26:391-4. doi: 10.1089/cap.2015.0091.

16. Lytle S, McVoy M, Sajatovic M. Long-acting injectable antipsychotics in children and adolescents. J Child Adolesc Psychopharmacol 2017; 27:2-9. doi: 10.1089/cap.2016.0055 